\title{
Diseño de arquitecturas de información lineales para mejorar la accesibilidad web
}

\author{
Por Ramón Voces-Merayo
}

\begin{abstract}
Resumen: En los últimos años ha habido un gran avance en lo que se refiere a la accesibilidad web: han aparecido normativas y leyes, herramientas, tecnologías y directrices suficientes como para crear adecuadamente contenidos accesibles. Sin embargo, pese a todos estos esfuerzos, la Web sigue siendo en gran medida poco accesible. Se analizan las diferentes posibilidades técnicas para la implementación de sistemas de acceso lineal a los sitios web, unos sistemas que facilitan tanto su accesibilidad como su usabilidad y que a menudo son olvidados por sus creadores.

Palabras clave: Arquitectura de la información, Accesibilidad web, Usabilidad, Sistemas de navegación, Acceso lineal.

Title: Information architecture and web accessibility: the design of linear access to the web
\end{abstract}

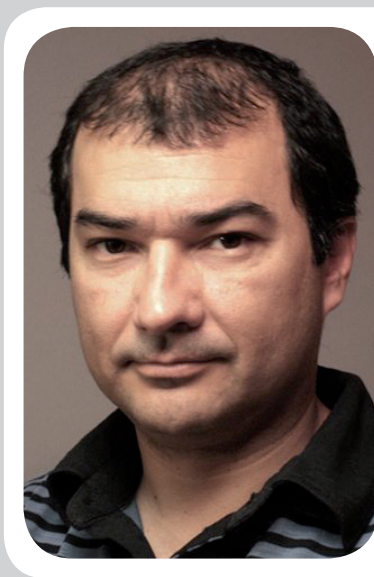
Ramón Voces-Merayo, doctor
en documentación e ingeniero
técnico en telecomunicacio-
nes, es profesor asociado del
Departamento de Comunica-
ción Audiovisual y Publicidad
II de la UAB y colaborador en
los estudios de información y
documentación de la UOC. Es
miembro del grupo de investi-
gación Laicom de la UAB y del
DigiDoc de la UPF. Sus líneas
de investigación se centran en
las tecnologías de la web, en
especial la accesibilidad y la
multimedia.

Abstract: Certainly, in recent years there has been a breakthrough in regard to web accessibility: policies and laws have emerged, along with tools, technologies and guidelines for creating accessible content properly. However, despite these efforts, the Web is still largely inaccessible. In this paper we have analyzed the different technical options for implementation of systems of linear access to the websites, systems which greatly facilitate the accessibility and the usability of these pages and are often forgotten by developers.

Keywords: Information architecture, Web accessibility, Usability, Navigation systems, Linear access.

Voces-Merayo, Ramón. "Diseño de arquitecturas de información lineales para mejorar la accesibilidad web”. El profesional de la información, 2010, julio-agosto, v. 19, n. 4, pp. 374-381.

DOI: 10.3145/epi.2010.jul.06

\section{Introducción}

Cada vez es más frecuente encontrarnos con sedes web que ofrecen una gran cantidad de información y que, a pesar de ello y a primera vista, se pueden considerar como "bien diseñadas". Obviamente, no suele ser casual ni gratuito: detrás queda, entre otros, el trabajo de los arquitectos de la información que no escatiman esfuerzos en elaborar buenos esquemas organizativos, óptimas estructuras que relacionen sus nodos, esmerados sistemas de etiquetado y de búsqueda y, por supuesto, sistemas de navegación que den unidad a todo ello.

Una buena arquitectura de la información (IA) no se basa solamente en el contenido, sino en su capacidad para dar respuesta a los cambios de contexto y a la diversidad de usuarios que acceden al mismo.

Es bastante frecuente ver cómo determinados diseños (obviamente incorrectos) dejan de lado a unos determinados usuarios que tienen todo el derecho, tanto moral como legal, de acceder adecuadamente a los contenidos: los discapacitados. El objetivo de una bue- na IA no sólo debe ser obtener un sistema usable sino un sistema usable y accesible.

En lo que se refiere a la accesibilidad web es justo reconocer que, poco a poco, se ha ido consolidando como disciplina: se ha definido un marco normativo y legislativo razonablemente avanzado, se han mejorado técnicas y tecnologías y se han creado nuevas directrices de creación de contenido accesible. A pesar de ello, no es difícil encontrar estudios de accesibilidad ${ }^{1}$ que demuestran que la Web sigue siendo en gran medida inaccesible.

\section{"El objetivo de una buena IA no sólo debe ser obtener un sistema usable sino además accesible"}

Uno de los aspectos más sorprendentes es la facilidad con la que se podrían resolver muchas de las ba- 
rreras de accesibilidad. Sirva como ejemplo un estudio publicado por WebAIM en octubre de 2009 (WebAIM, 2009) en el que se detallan las dificultades con las que se encuentran los usuarios que se enfrentan a la Web con un navegador de voz. Más allá de algún aspecto metodológico que podría ser criticable (como la falta de control sobre los sujetos de investigación) sus resultados apuntan a que muchas de estas dificultades tienen que ver con la carencia de medios para el acceso lineal a la página, lo que provoca problemas de operabilidad. Indican también que desde el punto de vista técnico, tanto el diseño como la implementación de estos sistemas resultan extremadamente sencillos.

En este artículo se va a tratar el diseño de vías de acceso lineal en sedes web, conociendo en primer lugar la perspectiva de las personas que se ven obligadas a utilizar estos tipos de sistemas para posteriormente presentar los diferentes problemas y soluciones técnicas disponibles en la actualidad.

\section{El contexto}

En los últimos años se han producido avances importantes en accesibilidad web y, además, como concepto se ha difundido considerablemente entre todos aquellos que participan de una forma u otra en el elaboración de proyectos web. De este modo, hoy en día resulta relativamente sencillo observar webs en las que se ha invertido un cierto tiempo en la mejora de la accesibilidad.

Desgraciadamente, con frecuencia no se obtienen unos resultados adecuados y todavía se quedan lejos de lo que podría considerarse como óptimo. Sin duda, el principal problema radica en una implementación deficiente, en muchas ocasiones provocada por unos conocimientos excesivamente superficiales de los creadores del sitio web que normalmente se centran en los aspectos más técnicos y teóricos de la accesibilidad, y olvidan -o ignoran- otros más reales como el entorno y el modus operandi que utilizan las personas discapacitadas para navegar.

Desde el punto de vista de la accesibilidad y en lo que respecta a la navegación, seguramente las personas más desfavorecidas son aquellas que, o bien no son capaces de utilizar un dispositivo apuntador (como el ratón), o bien presentan serias dificultades para hacerlo. En este grupo, se incluyen todas las que tienen discapacidades visuales y también aquellas con una discapacidad motriz tal que no les permite hacer uso de este tipo de dispositivos.
La forma en la que navegan las personas normales es diametralmente diferente a como lo hacen las personas con discapacidad. Para las primeras, la Web se presenta como un sistema espacial, en dos dimensiones, que hace que sea fácil hojearla a lo largo y ancho. En cambio, para las segundas, el acceso se hace linealmente, en una única dimensión: en el caso de discapacidad visual se utiliza un lector de pantalla que va dictando el contenido (como Jaws o Window-Eyes); en el caso de la discapacidad motriz, mediante el tabulador, accionado a partir de una amplia variedad de dispositivos capaces de recoger cualquier acción física controlada de la persona.

\section{"El reto para la IA es ofrecer acceso lineal a la información que mejore la consulta para los usuarios discapacitados"}

Evidentemente, esta perspectiva cambia radicalmente la forma en la cual se percibe la información. Páginas web como la que se muestra en la figura 1, que desde el punto de vista de una persona sin discapacidad podría considerarse como "bien diseñada", se convierten en una verdadera barrera para un discapacitado puesto que debe esperar hasta 2 minutos ${ }^{2}$, o apretar hasta 80 veces el tabulador para llegar a la sección del contenido. Además, atendiendo a las normas de coherencia de la usabilidad, esta barrera se repite en cada una de las páginas del sitio, haciendo que la experiencia final de usuario sea realmente frustrante.

Por lo tanto el reto para los arquitectos de la información consiste en ofrecer un sistema de acceso lineal a la información que mejore, en la medida de lo posible, la experiencia a estos usuarios.

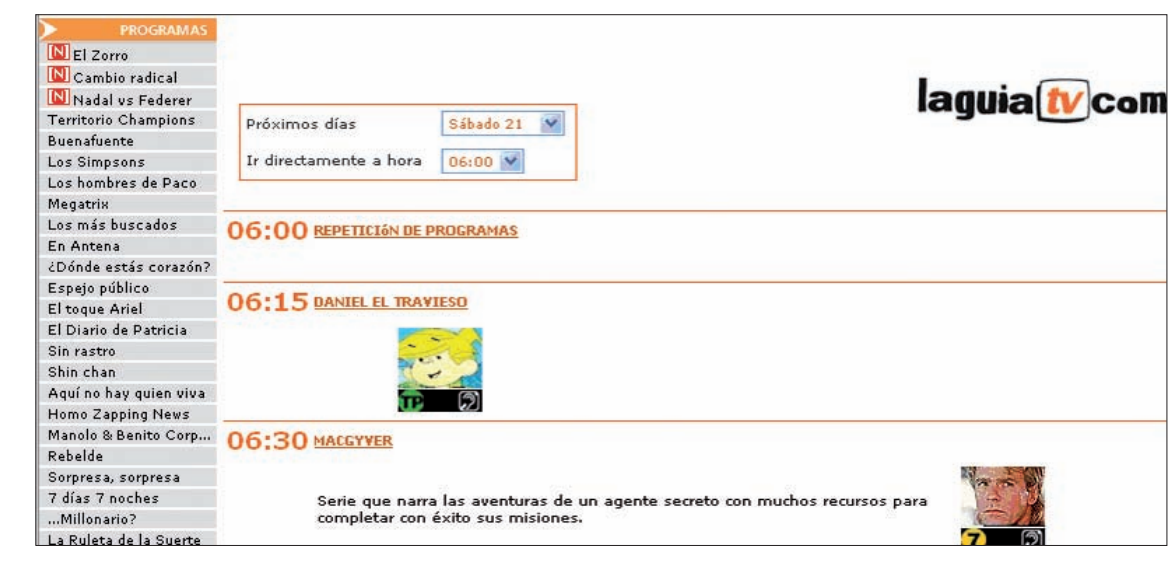

Figura 1. Web "aparentemente" bien diseñada 


\section{Diseño de acceso lineal a la información}

Este tipo de sistemas no suponen una novedad. Por ejemplo, una guía telefónica o un dvd audiovisual son productos habituales que tienen una concepción claramente lineal.

Si se analiza la forma de consulta de este tipo de productos, podremos llegar a la conclusión de que tienen dos características principales:

- Diseño secuencial. La información se presenta siguiendo un orden conocido que permite al usuario determinar su posición dentro del sistema de información y, a la vez, predecir cuál es el estado anterior y posterior. Por ejemplo, la guía telefónica se estructura mediante un esquema organizativo alfabético y un dvd mediante la numeración de escenas.

- Sistema de acceso aleatorio. De una forma u otra, se crea un índice que permite acceder directamente a un punto concreto del conjunto de información. De este modo, una guía telefónica presenta unos índices (alfabéticos, de actividades...) y un dvd un menú principal de escenas.

Como se verá a continuación, en lo que respecta a la web, construir un sistema de acceso lineal a un sitio web consistirá simplemente en adaptar las técnicas anteriormente descritas a las características particulares de este entorno.

\section{"Si la disposición de la información es lineal hay que determinar la secuencia según la cual se va a acceder a ella"}

\section{Diseño secuencial en una web}

Cuando la perspectiva de una página es lineal, es muy importante determinar la secuencia mediante la cual se va a acceder a la información.

Desgraciadamente, en una web el diseño del orden de la información puede ser bastante más complejo que en los casos anteriores. La razón fundamental es que en un sitio web se deben tener en cuenta dos tipos de orden:

- Orden de los elementos estructurales. Desde el punto de vis- ta estructural cada web se diseña mediante una serie de bloques, como por ejemplo, la cabecera, el pie, el sistema de navegación global, el contenido principal, etc., que, evidentemente, se deben presentar en un orden lógico.

- Orden de los contenidos informativos complejos. Las webs pueden incluir algunos elementos que presenten informaciones complejas como por ejemplo y sobre todo, las tablas y formularios. Obviamente, una secuenciación incorrecta de este tipo de contenidos puede provocar un gran problema de inteligibilidad.

\subsection{Orden de los elementos estructurales}

Existen dos posibilidades para crear la estructura de una web: por un lado utilizando las hojas de estilo css (conjuntamente con otras etiquetas html como div); y por otro, utilizando tablas, un sistema que ya se considera obsoleto y no recomendable.

Lo cierto es que, tanto en el primer caso como en el segundo, determinar a priori el orden de lectura de una web puede llegar a ser una tarea difícil. Por ello, más que evaluar directamente el código html y/o css, se suele utilizar algún tipo de aplicación que revele automáticamente el orden. En este sentido resulta especialmente útil cualquier lector de pantalla, la aplicación online Wave, el navegador textual Lynx o la barra de accesibilidad Accessible information solutions (AIS), que se utiliza en la figura 2.

\section{http://wave.webaim.org/}

Arreglar un error en el orden de lectura implica necesariamente modificar el código html. En general, si la estructura de la página se ha creado mediante css no suele ser un gran problema. En cambio, si se han utilizado tablas, podría llegar a ser costoso.

Respecto al orden que se debe seguir a la hora de secuenciar una web, no hay ningún estándar, ni siquiera de facto. Suele ser habitual, presentar la información de cada bloque de izquierda a derecha y de arriba a abajo. En el caso de que exista un sistema de búsqueda se podría considerar una buena práctica ubicarlo en las primeras posiciones de la secuenciación.

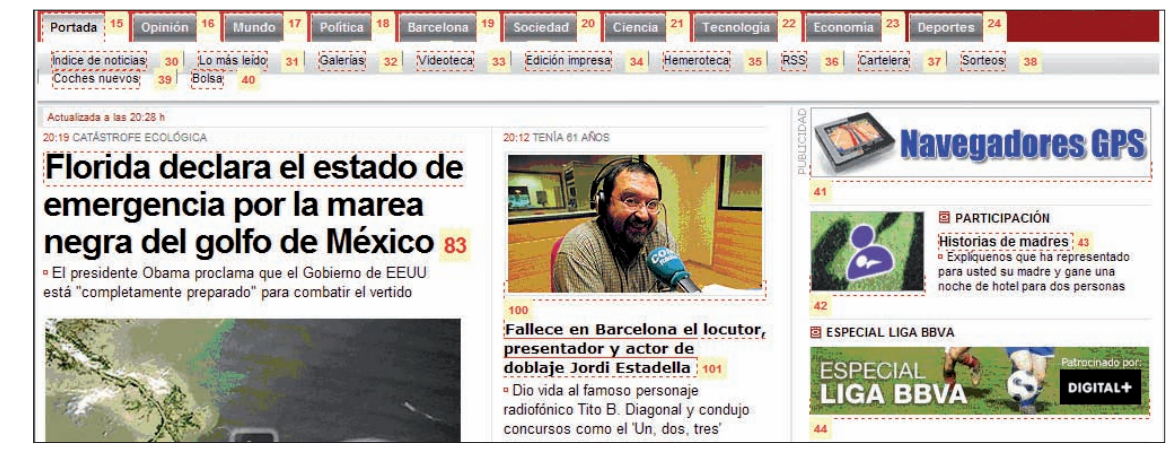

Figura 2. Ejemplo de utilización de la barra de accesibilidad AIS 


\subsection{Orden de los contenidos informativos complejos}

A la hora de construir el contenido, a menudo aparecen determinados elementos complejos que requieren también un diseño de secuenciación correcto. En concreto dos de ellos requieren una atención especial y conviene estudiarlos por separado: las tablas de datos y los formularios.

\section{Tablas de datos}

Representan el método más habitual de presentar informes estructurados en forma de matriz como por ejemplo los horarios de trenes o los índices bursátiles. Es evidente que su ecosistema natural y óptimo son los sistemas espaciales, pero esto no significa que no se pueda hacer un diseño lineal que permita un acceso cómodo a la información.

En el caso de las tablas el reto no sólo consiste en facilitar la secuenciación correcta de la información sino que, además, todos los datos (celdas) queden asociados a un identificador de columna y a un identificador de fila (WebAIM, 2009; Slatin; Rush, 2002, pp. 90-101). Por poner un ejemplo, en la tabla de la figura 3 , de nada serviría acceder al dato $10.421,30$ si no es posible asociarlo al último valor (identificador de columna) del Ibex 35 (identificador de fila).

Evidentemente, el primer objetivo de diseño a tener en cuenta es conseguir la máxima simplicidad, evitando en la medida de lo posible, modificar la distribución de filas y columnas dentro de la misma tabla.

Para comprobar el orden de secuenciación se puede utilizar de nuevo la barra AIS. En la figura 4 se observa cómo, en este caso, el orden establecido en la tabla de índices bursátiles es correcto.

La asociación entre el dato y su identificador de fila y/o columna, es una tarea que se debe ejecutar modificando el código html. Éste incluye tres atributos (scope para las tablas simples e id y headers para las más complejas) para resolver este problema. Para comprobar si todo es correcto, la mejor opción es utilizar un lector de pantalla.

\section{Formularios}

Desde el punto de vista procedimental los formularios representan un elemento esencial de interacción puesto que es el único sistema que permite el envío de datos por parte del usuario al servidor web $y$, en consecuencia, de poder acceder a una buena parte de servicios de valor añadido.

En cuanto accesibilidad, los formularios comparten algunas características comunes con las tablas (de hecho, muchos de ellos se maquetan haciendo uso de las mismas) pero presentan algunas dificultades adicionales por el hecho de que el usuario, además de recibir información, deberá introducirla.

Los factores principales a tener en cuenta son la titulación y orden de secuenciación de los objetos de formulario y el acceso por teclado (WebAIM, 2009).

Respecto al primer punto, todos los campos de los formularios deben ir siempre acompañados de un texto identificativo que indique qué datos acoge (nombre, apellido, DNI...). Además deben reproducirse antes o en el mismo momento en que el objeto sea enfocado.

Si no se diseña correctamente puede darse el caso de que, por ejemplo, se secuencien primero todas las etiquetas y después todos los objetos de formulario (Thatcher, 2006, pp. 142-143). De esta forma se hace prácticamente imposible asociar cada etiqueta con su objeto y, por lo tanto, conocer qué datos hay que poner.

Por otro lado, se debe asegurar el acceso por teclado. A menudo se incluyen determinados scripts que obligan a que el usuario utilice un ratón para introducir sus datos.

\begin{tabular}{|c|c|c|c|c|c|}
\hline & & & & & ACTUALIZAR \\
\hline \multicolumn{6}{|l|}{ INDICES BURSATTILES } \\
\hline IIESPAÑA & Último & Dif. & $\%$ Dif. & & \\
\hline IBEX 35 & $10.421,30$ & $-19,7$ & $-0,2 \%$ & $16: 26$ & Acciones \\
\hline IBEX MEDIUM CAP & $10.229,10$ & $-7,2$ & $-0,1 \%$ & $16: 25$ & Acciones \\
\hline IBEX SMALL CAP & $7.148,30$ & $-27,2$ & $-0,4 \%$ & $16: 24$ & Acciones \\
\hline FTSE LATIBEX ALL SHARE & $3.747,10$ & $-36,6$ & $-1,0 \%$ & $15: 55$ & Acciones \\
\hline \multicolumn{6}{|l|}{ MDO. CONTINUO } \\
\hline \pm EUROPA & Último & Dif. & $\%$ Dif. & & \\
\hline EUROSTOXX50 & $2.820,16$ & $-8,0$ & $-0,3 \%$ & $15: 40$ & Acciones \\
\hline FTSE 100 (Reino Unido) & $5.545,80$ & $-72,0$ & $-1,3 \%$ & $16: 10$ & Acciones \\
\hline DAX (Alemania) & $6.093,46$ & $-51,5$ & $-0,8 \%$ & $16: 10$ & Acciones \\
\hline CAC 40 (Francia) & $3.819,89$ & $-20,7$ & $-0,5 \%$ & $15: 55$ & Acciones \\
\hline FTSEMIB (Italia) & $21.647,24$ & $-48,5$ & $-0,2 \%$ & $15: 55$ & Acciones \\
\hline S\&P EUROPE 350 & $1.145,10$ & $-1,8$ & $-0,2 \%$ & $15: 55$ & \\
\hline \multirow[t]{2}{*}{ DOW JONES EUROPA } & $9.289,21$ & $-183,3$ & $-1,9 \%$ & $9 / 9 / 2008$ & \\
\hline & & & & & es Europeos \\
\hline
\end{tabular}

Figura 3. Tabla de datos

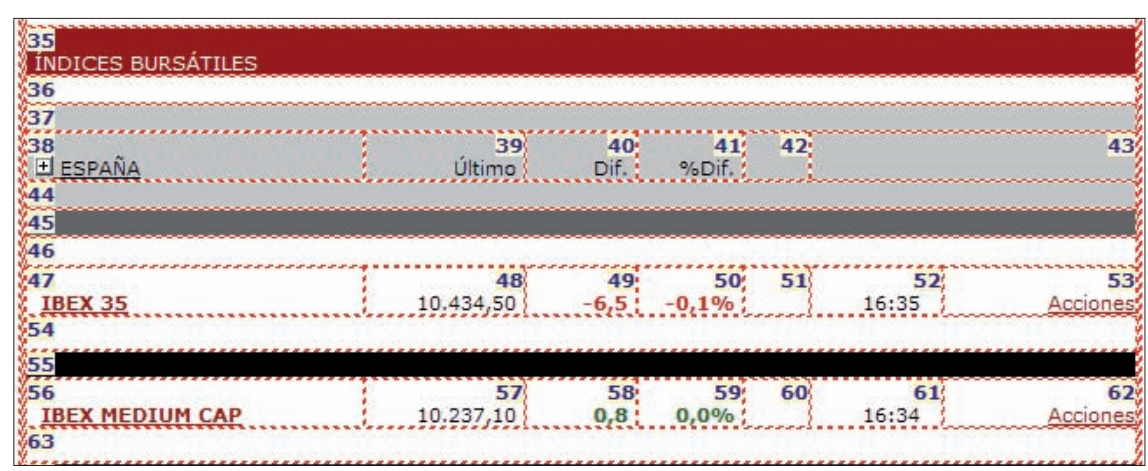

Figura 4. Utilización de la barra AIS en una tabla de datos 


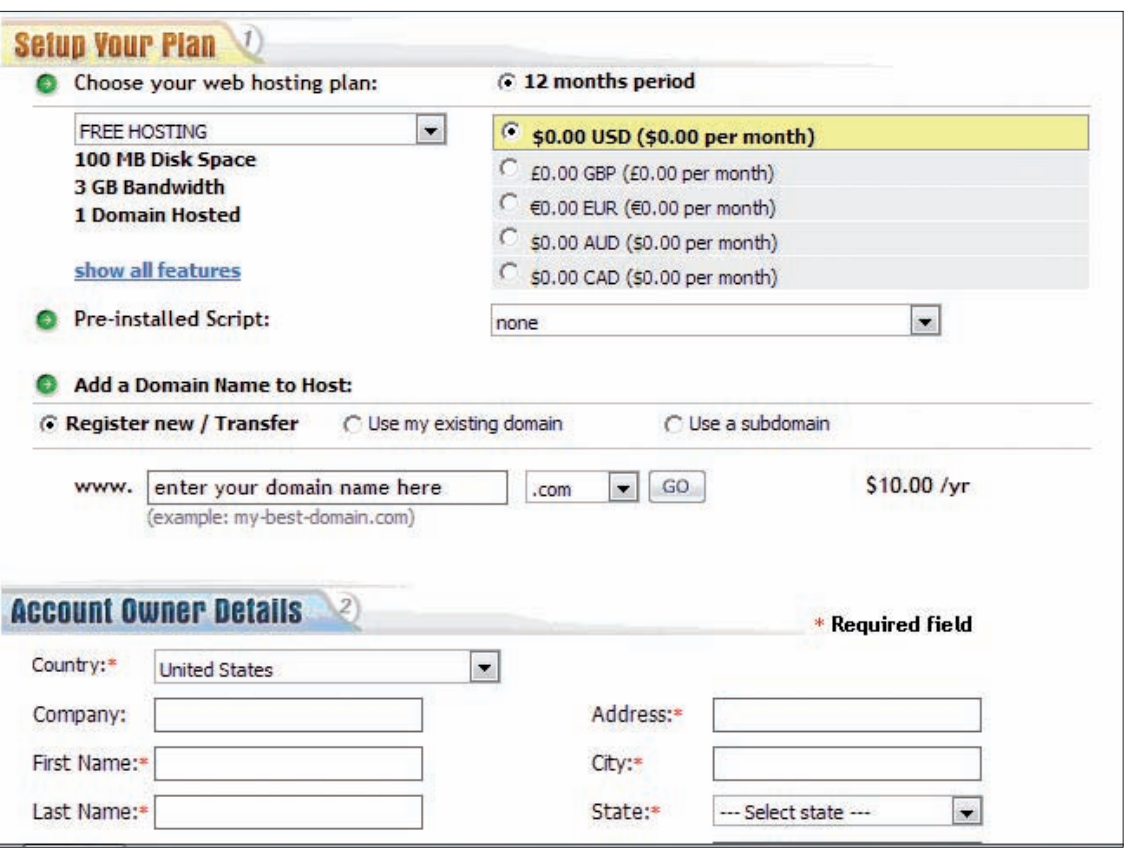

Figura 5. Formulario

La mejor manera de comprobar el funcionamiento correcto de un formulario es interactuando con él haciendo uso únicamente del teclado y ayudándose de alguna de las herramientas ya utilizadas con anterioridad, especialmente, un lector de pantalla.

\section{Diseño de sistemas de acceso aleatorio}

Uno de los objetivos de diseño más perseguidos en la elaboración de las sedes web es que sean coherentes. Esto implica mantener en la misma posición, tamaño y contenido, algunos de los bloques de su estructura como, por ejemplo, la cabecera o el sistema de navegación global.

Desgraciadamente, en muchas ocasiones este principio de coherencia hace que las personas discapacitadas que acceden a la web de forma lineal deban pasar por todas estas estructuras comunes antes de llegar al contenido, y esto, página tras página.

Quizá el problema sea que el enfoque tradicional de la arquitectura de la información está orientado a la navegación inter-nodos, centrándose en el objetivo de que el usuario consiga llegar de la forma más fácil posible al nodo que necesita. Pero una vez allí, no suele crearse ninguna estructura de soporte complementaria para los usuarios que acceden al nodo de forma lineal.

Para solucionar esto lo único que se requiere es el diseño de un sistema de navegación intra-nodo, que se presente rápidamente al usuario y que le permita acceder a las diferentes secciones o servicios de la página.

A continuación se presentarán varias técnicas para la implementación de sistemas de navegación intranodo. En concreto se analizarán las siguientes:
- Sistemas intrínsecos

- Enlaces de atajo

- Teclas de acceso rápido

- Landmarks

\subsection{Sistemas intrínsecos}

Los navegadores por voz leen entre líneas los códigos html para facilitar la navegación de sus usuarios. Además de reproducir secuencialmente todos los contenidos, normalmente presentan la opción de enseñar la lista de enlaces y la lista de títulos que existen en el sitio web. De este modo, permiten un acceso directo a estos enlaces o títulos.

Estas listas implican un sistema de navegación intra-nodo que no requiere ningún tipo de añadido al código html (de ahí lo de intrínseco) y que es muy utilizado por los usuarios. Aun así, conviene tener presente una serie de cuestiones que se comentarán a continuación.

\section{Navegación intra-nodo por títulos}

La gran mayoría de los lectores de pantallas, y cada vez más los navegadores convencionales ${ }^{3}$, permiten la navegación por títulos.

Mediante este tipo de navegación se puede acceder de forma secuencial a cada uno de los títulos presentes dentro de la página web, de forma que se reduce enormemente el tiempo necesario para llegar a uno determinado.

\section{"Los enlaces deben cumplir una regla muy simple: el texto asociado debe indicar claramente su propósito"}

El único aspecto a tener en cuenta es que para que la navegación por títulos funcione es necesario que sean identificados como tales. En html se debe hacer uso de las marcas $\mathrm{Hx}$, donde la $\mathrm{x}$ es un número que identifica el orden del título. Así H1 sería el título principal, H2 el subtítulo primero y así sucesivamente.

El problema es que muchos desarrolladores desconocen esta posibilidad y marcan los títulos con otras etiquetas, lo que dificulta este tipo de navegación. 


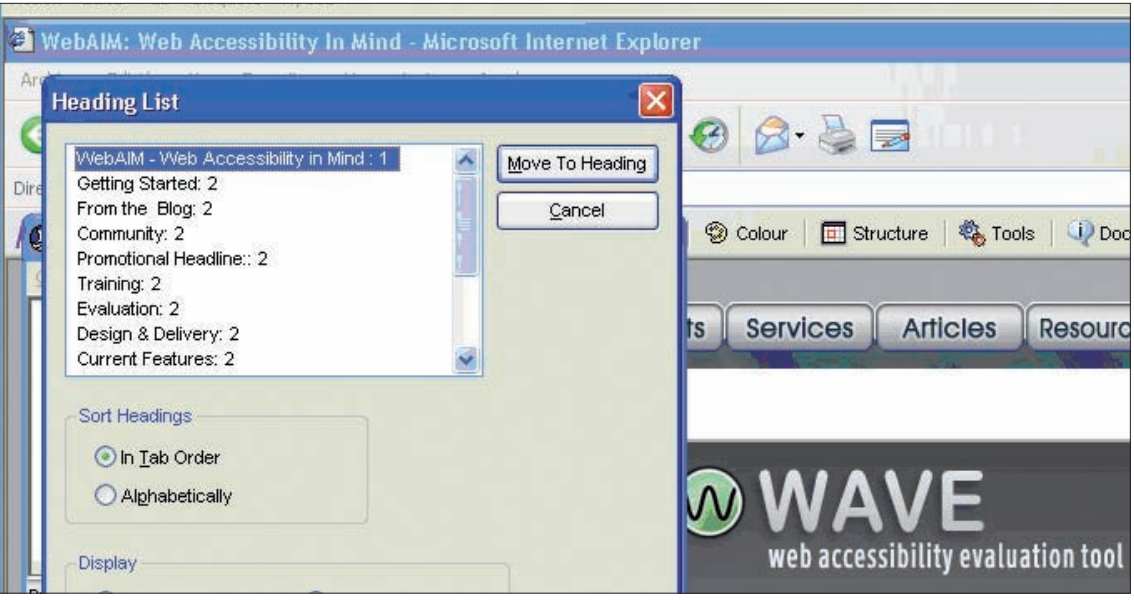

Figura 6. Ejemplo de identificación del orden de los títulos

Existen diversas técnicas de implementación que, básicamente, se diferencian en la forma en la que se presentan. Podemos encontrar tres tipos:

- Visibles. Aparecen en la interfaz como un enlace más.

- Invisibles. Son enlaces que se ocultan en la interfaz y sólo se pueden apreciar con un navegador de texto o un lector de pantalla. El problema de estos enlaces es que también son invisibles para las personas con discapacidades motrices.

\section{Navegación intra-nodo por enlaces}

Muchos usuarios con discapacidades físicas y visuales navegan directamente accediendo a los enlaces. Los primeros apretando la tecla de avance correspondiente, por ejemplo tab en Internet Explorer, y los segundos solicitando a su lector de pantalla la lista de enlaces de la página, por ejemplo ins+F7 en Jaws.

Desde el punto de vista de la accesibilidad, y especialmente para las discapacidades visuales, los enlaces deben cumplir una regla muy simple: en el texto asociado al enlace se debe indicar claramente su propósito. Esto es, antes de ser activado el usuario debe conocer con claridad lo que va a pasar a continuación.

Aunque parece una apreciación trivial, lo cierto es que la Web está repleta de ejemplos que no cumplen con esta regla. Existe multitud de enlaces con textos como clic aquí, probar, comprar, etc., que dificultan la navegación, puesto que en muchos casos los usuarios no son capaces de saber qué va a pasar cuando hagan clic aquí, qué van a probar o qué van a comprar.

En la figura 7 podemos ver un ejemplo bastante habitual: hay una serie de enlaces principales (consíguelo gratis y suscríbete ahora) y otros más secundarios (ambos con el mismo nombre: más información) que no aportan suficiente información sin el contexto en el que se muestran, de modo que utilizando este tipo de navegación no sería posible interactuar adecuadamente con esta página.

\subsection{Enlaces de atajo}

Son la forma más habitual de navegación intra-nodo. La implementación es muy simple: son enlaces convencionales que permiten un acceso directo a una sección determinada del sitio web y que, normalmente, se sitúan en la parte superior de la página para que su orden de lectura sea prioritario.
En la figura 9 se puede observar una serie de enlaces con el navegador Lynx que no aparecen en un navegador normal (en este caso Internet Explorer).

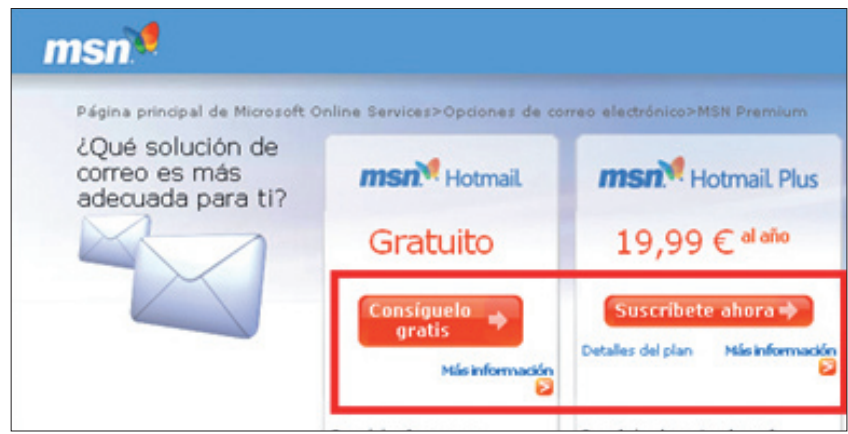

Figura 7. Enlaces que no indican claramente su propósito

- Emergentes. Son enlaces inicialmente invisibles pero que incorporan un código en JavaScript que los hace visibles en pantalla cuando se utiliza el teclado. De esta forma son igualmente accesibles por personas con discapacidades visuales y con discapacidades físicas.

http://www.jimthatcher.com/

\section{"Los enlaces de atajo permiten \\ un acceso directo a una sección determinada de la página"}

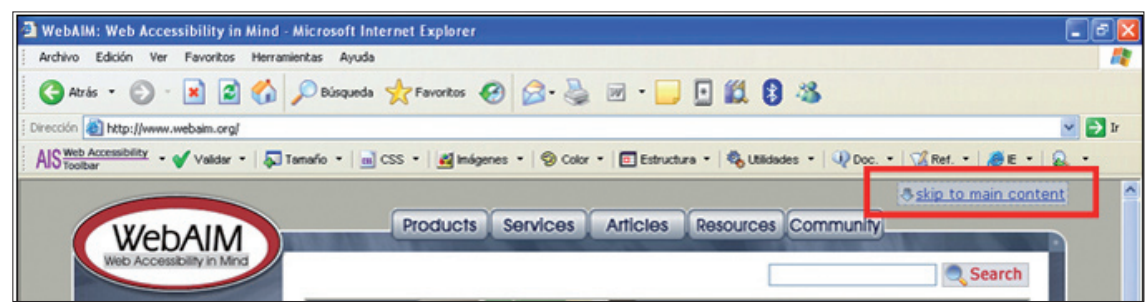

Figura 8. Enlace de atajo visible 


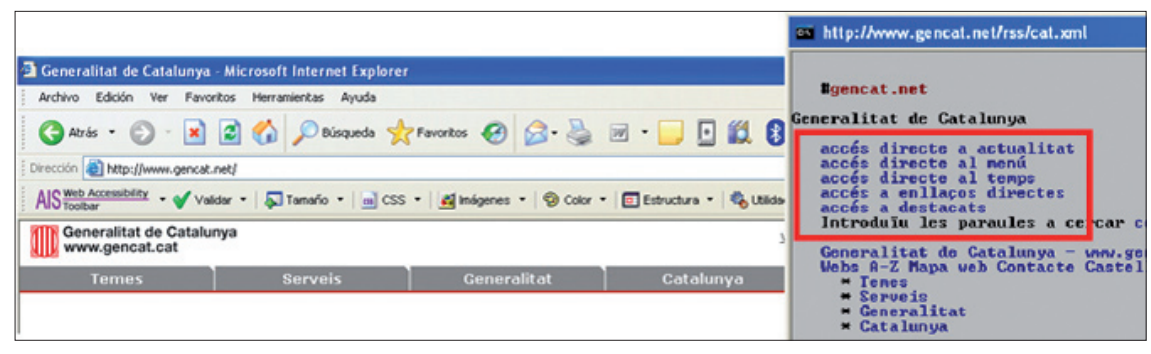

Figura 9. Enlaces de atajo invisibles

\subsection{Teclas de acceso rápido}

Son un atributo html (llamado accesskey) aplicable a diferentes etiquetas, en especial los enlaces, y permite establecer una conexión directa a la etiqueta que lo contiene.

Este atributo ha provocado grandes controversias ya que, a pesar de que conceptualmente puede ser una gran herramienta de acceso aleatorio, está implementada de formas muy diferentes en función del navegador y del lector de pantalla que se utilice.

Además existe la posibilidad de introducir conflictos (mismas teclas de acceso rápido) entre la web, el navegador, el lector de pantalla y el sistema operativo.

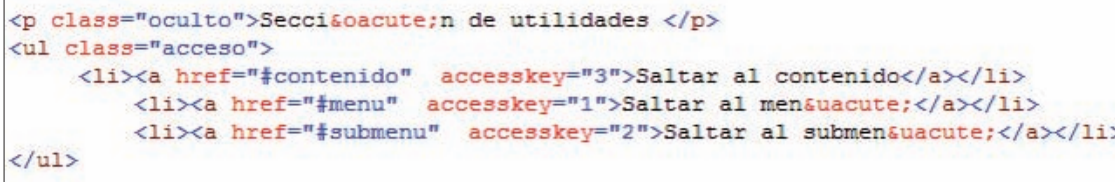

Figura 10. Accesskey

Aun así su uso sigue siendo bastante frecuente, y en muchas ocasiones se le asocian códigos numéricos que suelen ser los que reportan menos conflictos.

\subsection{Landmarks}

Representan el sistema de navegación intra-nodo más novedoso. De hecho están definidos en las WAIARIA (Web accessibility initiative-accessible rich internet applications) (W3C, 2009) una especificación del $W 3 C$ que todavía está en elaboración. A pesar de ello se está apostando por su uso y ya lo están soportando algunos lectores de pantalla y navegadores.

La idea es una evolución de los enlaces de atajo: en lugar de crear unos enlaces que accedan a cada sección, será cada sección la que se etiquete con un determinado rol. Estos roles están absolutamente definidos y recogen toda la casuística posible (banner, main, navigation, search...). Además, proporcionan un sistema semántico coherente para toda la web.

Cada landmark se activa mediante teclas de acceso rápido, que pueden proporcionar los lectores de pantalla y los navegadores convencionales. En la figura 11 se puede observar la lista de landmarks en Firefox con el complemento Accessibility Extension.

\section{"Ninguno de los métodos presentados comporta grandes requerimientos técnicos, ni elevados tiempos de implementación y, en consecuencia, coste económico"}

\section{Conclusiones}

Pese a los avances de los últimos años, la Web sigue siendo en gran medida poco accesible. Se podrían buscar diferentes motivos, pero parece plausible apuntar a la falta de conocimientos de los desarrolladores de webs como fundamental (Voces-Merayo; Codina, 2008).

En este artículo se han analizado las diferentes posibilidades técnicas para la implementación de sistemas de acceso lineal a sitios web, que facilitan tanto la accesibilidad como la usabilidad de las páginas.

Ninguno de los métodos presentados comporta grandes requerimientos técnicos, ni elevados tiempos de implementación y, en consecuencia, coste económico. No obstante, todavía son excesivamente pocas las sedes web que los incorporan.

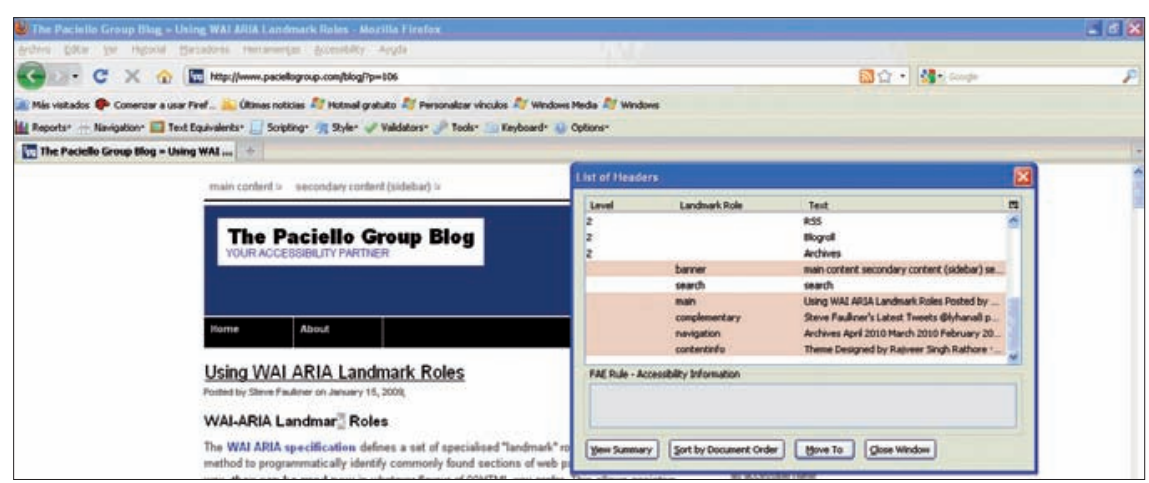

Figura 11. Lista de landmarks en Firefox 
Es cada vez más importante, moral y legalmente necesario posicionar a la accesibilidad como un requisito de obligado cumplimiento para todas las sedes web.

\section{Notas}

1. Son especialmente interesantes los estudios realizados por el Observatorio de accesibilidad de Discapnet, los del Cermi o el reciente trabajo de la UE titulado Web accessibility in European countries: level of compliance with latest international accessibility specifications, notably WCAG 2.0, and approaches or plans to implement those specifications.

http://www.discapnet.es/Castellano/areastematicas/Accesibilidad/Observa torio_infoaccesibilidad/informesInfoaccesibilidad/Paginas/default.aspx http://www.cermi.es/ES-ES/BIBLIOTECA/Paginas/Inicio.aspx http://ec.europa.eu/information_society/activities/einclusion/library/ studies/web_access_compliance/index_en.htm

2. A velocidad estándar de Jaws

3. En el navegador Opera las teclas s y w permiten pasar al título siguiente o al anterior respectivamente.

\section{Bibliografía citada}

Slatin, John M.; Rush, Sharron. Maximum accessibility: making your web site more usable for everyone. Addison-Wesley professional, 2002. ISBN 978-0201774221.

Thatcher, Jim et al. Web accessibility. Web standards and regulatory compliance. Friends of ED, 2006. ISSN 978-1-59059-638-8.

Voces-Merayo, Ramón; Codina, Lluís. "La accesibilidad potencial y real del formato pdf: análisis de diarios digitales españoles". El profesional de la información, 2008, v. 17, n. 2, pp. 205-212.

W3C. Accessible rich internet applications (WAI-ARIA) 1.0.

http://www.w3.org/TR/wai-aria/

WebAIM. Creating accessible forms. http://www.webaim.org/techniques/forms/

WebAIM. Creating accessible tables. http://www.webaim.org/techniques/tables/

WebAIM. Screen reader user survey results. http://webaim.org/projects/screenreadersurvey2/

Ramón Voces-Merayo. Universitat Autònoma de Barcelona.

ramon.voces@uab.cat

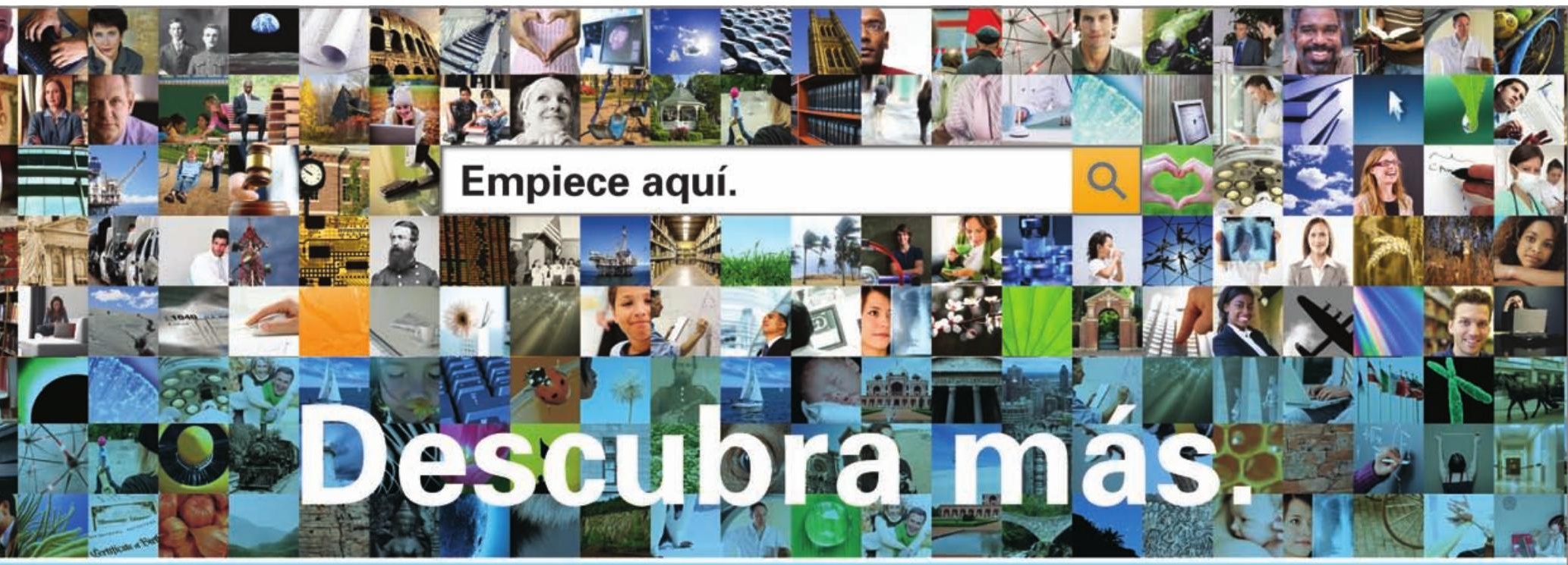

Nuestia boderosa herramienta de busqueda le dara acoeso a todo un mundo bor descubrir Su opinion nos ha ayudado a construir la nueva Plataforma ProQuest, renovada desde sus cimientos, que incorpora todas las funcionalidades mas valoradas por nuestros usuarios v opciones innovadoras Todo esto está en la nueva plataforma ProQuest. Y su lanzamiento será en 2010.

Para más información sobre nuestra nueva plataforma ProQuest, visite www.proquest.com/go/yourpathcontacte con nosotros en platform@proquest.co.uk indicando la referencia AD 69710. 


\section{masmedios}

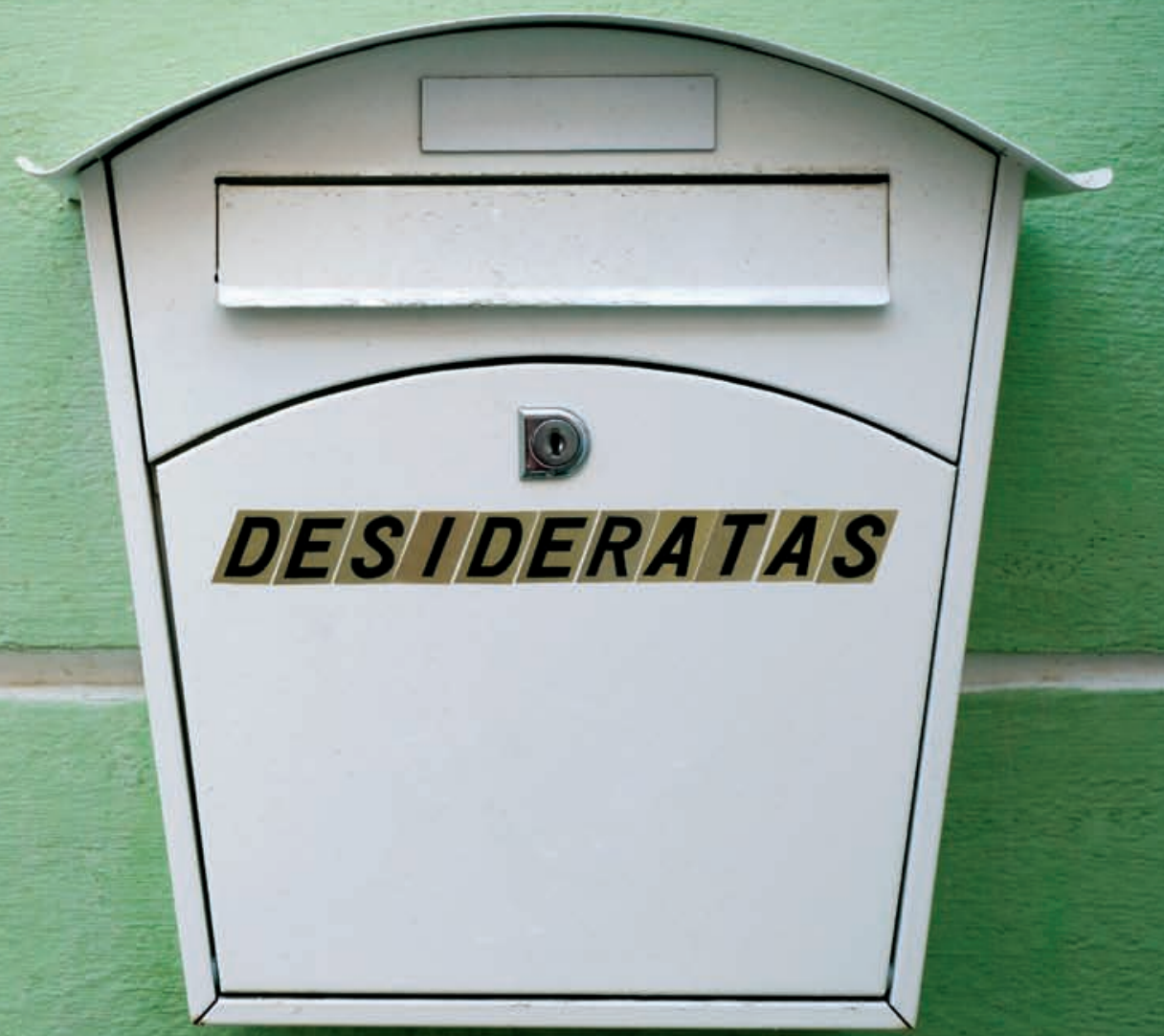

Te entendemos 99

Sistemas de gestión de bibliotecas Open Source Interfaces interactivas y OPACs Repositorios OAI

Gestión documental y de archivos

Digitalización

Outsourcing de servicios documentales

Desarrollo de sitios web / multimedia / e-learning

Comunicación y e-marketing de servicios de información
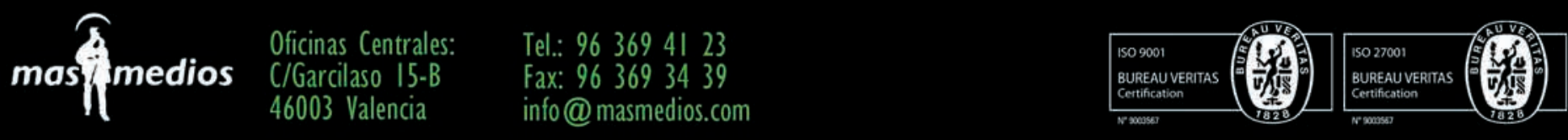

www. masmedios.com 